Felix Bildhauer/Eric Fuß/Sandra Hansen-Morath/ Franziska Münzberg (Mannheim)

\title{
Starke und schwache Adjektivflexion in neuem korpuslinguistischen Licht
}

\begin{abstract}
In Adjektivreihungen ohne Determinierer (in neuem korpuslinguistisch-em/ -en Licht) und in Fügungen aus Pronominaladjektiv und attributivem Adjektiv (mancher ausbildend-er/-e Betrieb) treten Schwankungen zwischen Parallel- und Wechselflexion auf, die von einem komplexen Zusammenspiel verschiedener grammatischer und außergrammatischer Faktoren beeinflusst werden. Auf der Basis einer explorativen Korpusstudie werden im vorliegenden Beitrag zunächst einschlägige Einflussgrößen identifiziert und deren Effektstärken geschätzt. Im Anschluss wird gezeigt, dass entgegen bisherigen Annahmen nach Pronominaladjektiven keine allgemeine Tendenz zur schwachen Flexion vorliegt, sondern mit Ausnahme des Kontextes Dat. Sg. Mask./Neutr. diachron eine Ausbreitung der Parallelflexion (stark/stark) beobachtbar ist.
\end{abstract}

\section{Korpuslinguistische Methoden}

Dieser Beitrag präsentiert zwei explorative Korpusstudien zur Adjektivflexion. Beide Fallstudien basieren auf Korpora des Instituts für Deutsche Sprache. In erster Linie werden dabei Ausschnitte verschiedener Größe (ca. 7 Mrd. bzw. ca. 122 Mio. Tokens) des Deutschen Referenzkorpus DeReKo (Kupietz et al. 2010) verwendet, die hinsichtlich bestimmter Dokumentmetadaten (z.B. Land, Region, Medium) vervollständigt und stratifiziert wurden (Bubenhofer/Konopka/Schneider 2014, Kap. 1). Darüber hinaus greift die zweite Studie auf historische Daten des HISTArchivs zurück, die in ihrer großen Mehrheit aus dem 19. Jahrhundert stammen und für diesen Zweck neu aufbereitet und in einem Korpus von ca. 30 Mio. Tokens zusammengefasst wurden. ${ }^{1}$ Die Studien verwenden automatisierte Wortannotationen (Wortart, Lemma, morphologische Merkmale), erzeugt mit Standardwerkzeugen (TreeTagger, Schmid 1995; SMOR, Schmid/Fitschen/Heid 2004; MarMoT, Müller/Schmid/Schütze 2013) und einigen Anpassungen.

1 Vgl. www1.ids-mannheim.de/lexik/abgeschlosseneprojekte/historischeskorpus/historischeskorpus.html (Stand: 19.7.2018).

https://doi.org/10.1515/9783110622591-014 
Für die erste Fallstudie wurde eine relativ komplexe statistische Modellierung gewählt, u.a. ein hierarchisches generalisiertes lineares Modell mit logit-link (GLMM, entspricht hier einer logistischen Regression mit variablen Intercepts). Dabei wird die Wahrscheinlichkeit modelliert, in einem gegebenen Fall (d.h. abhängig von einer Reihe von Einflussgrößen) eine bestimmte Flexionsendung vorzufinden (starke vs. schwache Flexion).

Die verschiedenen potenziellen Einflussgrößen wurden teils auf Grundlage vorhandener Literatur ausgewählt, teils entsprechen sie eigenen Hypothesen. An dieser Stelle soll betont werden, dass sich beide Studien zunächst als explorativ verstehen: $p$-Werte werden vorläufig als Maß für Evidenz interpretiert, und insbesondere erfolgt kein Entscheidungstest. Dies ist geboten, weil für keine der potenziellen Einflussgrößen eine spezifische Alternativhypothese (d.h. mit erwarteter Effektstärke) formuliert werden kann. Darüber hinaus sind auch einige ursprünglich berücksichtigte Einflussgrößen nicht mehr in den unten präsentierten Modellen enthalten, sodass die selegierten Modelle streng genommen nicht mehr die Voraussetzungen für übliche inferenzstatistische Verfahren erfüllen (siehe Berk/ Brown/Zhao 2010; Selvin/Stuart 1966).

Ein letzter Vorbehalt betrifft die üblichen Schwierigkeiten von Studien, die auf Basis großer, relativ heterogener Korpora durchgeführt werden, so u.a.:

a) Aufgrund der Korpuszusammensetzung und -aufbereitung lässt sich i.d.R. nur Variation innerhalb der Textmenge untersuchen, jedoch nicht Variation innerhalb aller Texte desselben Verfassers. Daten, die von potenziell unterschiedlichen, individuellen Grammatiken „erzeugt“ wurden, vermischen sich dabei.

b) Dies kann zu Abhängigkeiten in den Daten führen (mehrere Datenpunkte pro Verfasser, aber gerade in Zeitungstexten sind diese oft nicht namentlich erfasst), die wiederum Voraussetzungen vieler inferenzstatistischer Verfahren verletzen, wenn sie nicht berücksichtigt werden.

c) Solche Verfahren erfordern zudem echte Zufallsstichproben aus wohldefinierten Grundgesamtheiten (siehe Berk/Freedman 2009). Demgegenüber müssen viele Korpora wohl als convenience samples gelten.

Vor diesem Hintergrund sind die hier präsentierten korpuslinguistischen Befunde als tentativ zu betrachten. Sie können als Ausgangspunkt für weitere, auch konfirmative Studien dienen, indem sie wertvolle Anhaltspunkte z.B. für Effektstärken und Modellspezifikationen liefern. Dies ermöglicht wiederum die gebotenen korpuslinguistischen Replikationen und auch experimentelle Validierungen unter kontrollierten Bedingungen, in der Hoffnung auf konvergierende Befunde. 


\section{Fallstudien zur Adjektivflexion}

Eine Besonderheit des Deutschen ist, dass attributive Adjektive nicht nur nach Kasus, Numerus und Genus flektiert werden, sondern zusätzlich stark oder schwach. Dabei lässt sich besonders in redigierten Texten eine Regularität beobachten:

Folgt ein attributives oder nominalisiertes Adjektiv/Partizip auf einen Determinierer mit (immer starker) Flexionsendung, wird es schwach dekliniert (diese blauen Blüten), sonst stark (blaue Blüten); vgl. u.a. Dudengrammatik (2016, S. 368). ${ }^{2}$ Von dieser Regularität gibt es im standardnahen Deutsch drei Ausnahmen:

1. maskuline und neutrale Adjektive im Dativ Singular, die auf ein anderes attributives Adjektiv folgen (in neuem korpuslinguistischem/korpuslinguistischen Licht) - Schwankungen beim jeweils zweiten Adjektiv

2. Adjektive nach Wörtern, die sowohl Eigenschaften von Determinierern als auch Eigenschaften von Adjektiven haben (Pronominaladjektiven wie solche, manche) - Schwankungen beim jeweils zweiten Adjektiv

3. maskuline und neutrale Adjektive im Genitiv Singular (aufgrund schlechten Wetters) - starke Endung -s nur noch in pronominaler Flexion (unseres Schrebergartens)

Die beiden zuerst genannten Ausnahmen betreffen Schwankungen zwischen Parallelflexion (beide Adjektive erhalten die starke Endung) und Wechselflexion (das erste Adjektiv erhält die starke Endung, das zweite die schwache). Dieser Beitrag beschäftigt sich speziell mit diesen beiden Zweifelsfällen.

\subsection{Flexionsschwankungen im Dativ Singular Maskulinum und Neutrum}

Im Folgenden steht zunächst die Schwankung zwischen starker (1) und schwacher (2) Flexion im Dativ Singular Maskulinum und Neutrum im Fokus:

(1) Stark: Denn der Rückverweis an die streitenden Parteien ist der einzig mögliche Weg, um jeden Ruch des Staatsinterventionismus zu vermeiden - und mit sanftem, indirektem Druck diese leidige, politisch kontraproduktive Affäre beizulegen. (die tageszeitung, 22.4.2004, S. 11)

2 Die „Tendenz, die Merkmale nur noch an einem einzigen Wort der Nominalphrase anzuzeigen“ (Dudengrammatik 2016, S. 954), wird als Monoflexion bezeichnet. 
(2) Schwach: Das Gericht hat trotz fehlendem direkten Beweis keine Zweifel an der Schuld des Angeklagten. (Die Südostschweiz, 9.6.2012, S. 7)

\subsubsection{Erklärungsansätze}

Dass in artikellosen Nominalphrasen mit mehr als einem attributiven Adjektiv die schwache Endung -en anstelle der starken Endung -em stehen kann, wird teilweise unter „phonologische $m$-Schwäche“ gefasst. Dagegen wendet Nübling (2011, S. 178) ein, dass eine rein phonologisch verursachte Präferenz für die schwache Endung auch das erste der Adjektive betreffen müsste, nicht nur das zweite. Würde es sich aber ausschließlich um einen Trend zur Monoflexion handeln, so bliebe unerklärt, warum ausgerechnet der Dativ Singular Maskulinum und Neutrum betroffen ist, nicht aber etwa der Genitiv Plural oder der Dativ Singular Femininum (vgl. Kubczak 2015). Plausibler scheint die Erklärung, dass hier phonologische, morphologische und syntaktische Einflüsse zusammenwirken und so nur beim zweiten Adjektiv zur $m$-Schwäche führen (siehe Gallmann 2016, S. 1). Auch könnten semantischsyntaktische Normen beim Schreiben und vor allem im Lektorat eine Rolle spielen (vgl. etwa Peter 2013, S. 202).

Dabei konkurrieren drei einander widersprechende Normen:

1. Nach Adelung (1782/2010, S. 634) wird in den obliquen Kasus grundsätzlich wechselflektiert; vgl. auch Behaghel (1923, S. 212, Fall b).

2. Nach Heuer/Flückiger/Gallmann (2017, S. 139) soll immer, auch in Fällen wie (2), parallel flektiert werden. Behaghel (1923, S. 212, Fall a) zufolge ist „dieser Typus [...] der durchaus herrschende, insbesondere in der Gegenwart“, also in den Zwanzigerjahren des 20. Jahrhunderts.

3. Nach der „Einschließungsregel“ (Nübling 2011, S. 179) kann - oder soll - nur dann wechselflektiert werden, wenn das erste Adjektiv die Gesamtheit aus zweitem Adjektiv und Nomen modifiziert, vgl. (2). Behaghel (1923, S. 212, Fall c) kommentiert: „Dieser Typus scheint überwiegend in Norddeutschland zu Hause zu sein.“

Die Einschließungsregel - nicht als Flexions-, sondern als Interpunktionsnorm - ist auch in der geltenden amtlichen Rechtschreibregelung (Rat für deutsche Rechtschreibung 2018, § 71 (2) E1) festgeschrieben: Ein Komma soll nur zwischen gleichrangigen Adjektiven wie in (1) gesetzt werden.

Damit erhält das Komma in Texten geübter Schreibender auf der grafischen Oberfläche eine ähnliche Funktion als Koordinierer wie in der syntaktischen Struktur eine Konjunktion. Tatsächlich wird rechtschreibdidaktisch der „und“-Test ein- 
gesetzt: Kann zwischen die beiden Adjektive ein und (oder seltener Wörter bzw. Wortgruppen wie also, und zwar, d.h.) gesetzt werden, ist ein Komma an dieser Stelle korrekt.

\subsubsection{Datengrundlage}

Um zu untersuchen, wie verschiedene mögliche Einflussgrößen (Prädiktoren) bei der Wahl zwischen starker und schwacher Flexion zusammenwirken, haben wir mit zwei leicht voneinander abweichenden Suchanfragen zwei unterschiedlich große Datensätze erzeugt.

Der größere der beiden Datensätze umfasst nach dem Aussortieren von Fehlbelegen noch über 119.000 Belegsätze. Gesucht wurde im größeren, ca. 7 Mrd. Tokens umfassenden Ausschnitt aus DEREKo nach einer Präposition, auf die ein Adjektiv mit der Endung -em direkt folgt. Optional kann darauf ein Komma oder eine Konjunktion (ggf. als Komma plus Konjunktion) direkt folgen. Direkt danach steht ein Adjektiv mit einer der Endungen -em oder -en, unmittelbar darauf ein Nomen.

Der kleinere Datensatz, erzeugt aus dem kleineren Teilkorpus von nur ca. 122 Mio. Tokens, enthält nach dem Aussortieren von Fehlbelegen knapp 1.600 Belegsätze zur Suchanfrage Präposition - optionales Adverb - erstes Adjektiv optionales Komma - optionales Adverb - zweites Adjektiv - Nomen.

\subsubsection{Belegannotation}

Beide Datensätze enthielten bereits eine Annotation nach Land, Region, Jahr und inhaltlicher Domäne. Um eventuelle Frequenzeffekte zu kontrollieren, wurde in beiden Datensätzen die DeReWo-Frequenzklasse (Lexemfrequenzen nach Belica et al. 2012) jeweils des ersten und des zweiten Adjektivs nachgetragen. ${ }^{3}$

Ebenfalls in beiden Datensätzen nachgetragen wurden die Silbenzahl des ersten und des zweiten Adjektivs und ob das Nomen auf - $m$ endet und wie viele $m$ es zwischen Anfangs- und Endbuchstaben enthält (Zählung von $\mathrm{mm}$ in beiden Fällen als 1). Die Hypothese war, dass beides starke Flexion des zweiten Adjektivs auslösen würde, $m$ in der Mitte des Nomens allerdings seltener als ein $m$ am Ende des Nomens, vgl. Niebuhr 2017).

3 Für die nachträgliche Belegannotation danken wir unseren Hilfskräften Anna Konovalova, Oskar Jabs und Nagehan Cetin. 
Der kleine Datensatz enthält zusätzlich die manuelle Annotation nach Genus des Nomens, Wortbildung der Adjektive (Partizip - lexikalisiertes Partizip - kein Partizip), semantischer Klasse der Adjektive (eine Synthese aus Eichinger 1987, 1991; Trost 2006; Nübling 2011; Dudengrammatik 2016) sowie „und“-Test.

\subsubsection{Auswahl an Ergebnissen}

Ein erster Blick auf die Zahlen im großen Datensatz stärkt die Vermutung, dass ein Komma als Prädiktor ähnlich - wenn auch nicht so verlässlich ${ }^{4}$ - wirkt wie eine Konjunktion: Bei Koordination mit und wird das zweite Adjektiv in nur 5\% der Belege schwach flektiert, bei Koordination mit Komma aber sind es $11 \%$, bei Koordination mit Komma (ohne eine Konjunktion) 21\% schwach flektierte zweite Adjektive; demgegenüber aber 65\%, wenn Anzeichen für Koordination fehlen, also bei direkt aufeinanderfolgenden Adjektiven.

Der Prädiktor Komma bzw. Koordination soll nun nicht isoliert, sondern mithilfe von Regressionsanalysen ${ }^{5}$ im Zusammenspiel mit anderen Einflussgrößen analysiert werden. Im großen Datensatz wie im kleinen Datensatz ist der Faktor „Land“ relevant: Die Beobachtung von Peter (2013, S. 201), dass in der Schweiz eher stark flektiert wird, können wir bestätigen.

Der Unterschied zwischen der Schweiz und den übrigen Ländern wird besonders bei Nominalgruppen ohne Komma sichtbar. Im an die Schweiz angrenzenden Südwesten Deutschlands, aber auch in Thüringen und Sachsen gibt es ähnliche Tendenzen wie in der Schweiz. ${ }^{6}$ Nur am großen Datensatz ist in der Regressionsanalyse eine Entwicklung über die Jahrzehnte (1900 bis 2014) ${ }^{7}$ hin zur schwachen Flexion ablesbar $(\mathrm{p}<0.001)$. Besonders in der Schweiz wird immer öfter schwach flektiert, es findet also eine Angleichung an Deutschland und Österreich statt.

4 Zu Grenzfällen zwischen Struktur (1) und Struktur (2) vgl. Moulin (2000, S. 87).

5 Prädiktoren im gemischten logistischen Regressionsmodell für den großen Datensatz: Konjunktion/Komma, inhaltliche Domäne, Land, Jahrzwanzigst, Silbenanzahl des zweiten Adjektivs, Frequenzklasse des ersten Adjektivs, $m$ am Ende des Nomens, $m$ in der Mitte des Nomens; Stamm des ersten Adjektivs als Zufallsfaktor (Intercept).

Prädiktoren im logistischen Regressionsmodell für den kleinen Datensatz: Land, „und“-TestErgebnis, Komma JA/NEIN, semantische Klasse des zweiten Adjektivs, Silbenanzahl des zweiten Adjektivs, Frequenz des ersten Adjektivs, $m$ am Ende des Nomens, $m$ in der Mitte des Nomens. 6 Beides wurde mit Assoziationsplots (vgl. Cohen 1980; Friendly 1992; Meyer/Zeileis/Hornik 2005) visualisiert.

7 Gut belegt sind allerdings nur die Jahre ab 1990. 
Überraschend sind die Ergebnisse zur semantischen Klasse des zweiten Adjektivs im kleinen Datensatz: Im multifaktoriellen Modell treten nur Ordinalzahladjektive $^{8}$ und (wesentlich weniger deutlich) Farbadjektive als schwach flektierend hervor (vgl. jedoch Schäfer 2017).

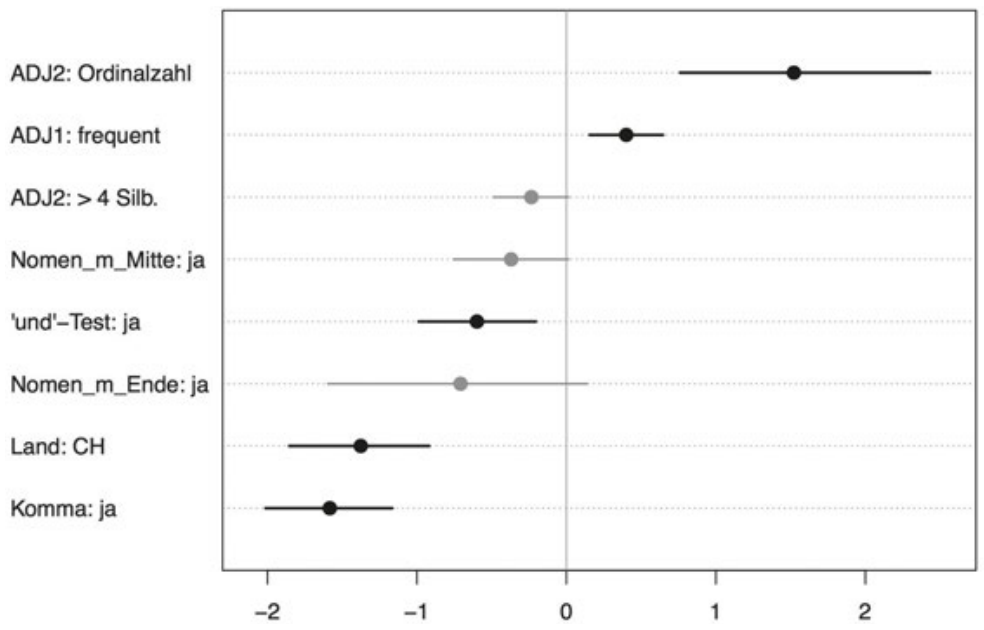

Abb. 1: Einflussgrößen zur Parallel-/Wechselflexion: geschätzte Koeffizienten. Positive Koeffizienten bedeuten eine erhöhte Wahrscheinlichkeit für Wechselflexion.

ADJ2: zweites Adjektiv, ADJ1: erstes Adjektiv.

ADJ1: frequent: DeReWo-Frequenzklasse des ersten Adjektivs $\leq 8$ (je häufiger das Lexem, desto niedriger die Frequenzklasse).

ADJ2: > 4 Silb.: Das zweite Adjektiv hat über 4 Silben (Flexionssuffix mitgerechnet). ${ }^{9}$ Nomen_m_Mitte, Nomen_m_Ende: $\boldsymbol{m}$ in der Mitte des Nomens bzw. $\boldsymbol{m}$ am Ende des Nomens. ${ }^{10}$ , und'-Test: Zwischen die beiden Adjektive lässt sich und oder etwas wie $\boldsymbol{d} . \boldsymbol{h}$., und zwar einfügen. ${ }^{11}$

Abbildung 1 zeigt geschätzte Koeffizienten aus einem logistischen Regressionsmodell $^{12}$ zum kleinen Datensatz. Je weiter ein Punkt von der senkrechten Mittellinie

8 Es handelt sich fast nur um Sportnachrichten (Muster: nach gewonnenem ersten Satz, nach glücklichem zweiten Tor).

9 Wie zu erwarten, ist diese Einflussgröße im großen Datensatz (ohne Umcodierung in einen binären Faktor) signifikant $(\mathrm{p}<0.001)$.

10 Beide Einflussgrößen sind im großen Datensatz signifikant $(\mathrm{p}<0.001)$.

11 In den Originaldaten 4 Werte: 0 (es lässt sich nichts einfügen), 1 (es lässt sich und einfügen), 2 (es lässt sich und zwar o.̈̈. einfügen), 3 (das Adjektiv wird nach einem Komma wiederholt). 1 und 2 sind hier zusammengeführt, 3 (ein Vorkommen) wurde nicht berücksichtigt.

12 Alle Prädiktoren wurden hierfür in binäre Faktoren umcodiert. 
(0) entfernt ist, desto stärker ist der Effekt. Nach links sind negative Koeffizienten abgetragen: Die Wahrscheinlichkeit für schwache Flexion des zweiten Adjektivs nimmt ab. Nach rechts sind positive Koeffizienten abgetragen, mit denen die Wahrscheinlichkeit für schwache Flexion steigt. Die waagerechten Linien stehen für 95\%-Konfidenzintervalle.

Das Komma erlaubt also im gegebenen standardnahen Korpus recht gute Vorhersagen - solange man sich auf die Interpretation des Kommas als Koordinationssymptom einlässt.

\subsection{Flexionsschwankungen nach Pronominaladjektiven}

\subsubsection{Einleitung}

Das Flexionsverhalten von Adjektiven nach sogenannten Pronominaladjektiven wie all-, solch-, welch-, einig-, sämtlich-, beid-, folgend- und ander-, also Elementen, die sowohl Eigenschaften von Determinierern als auch von Attributen aufweisen, ist ein von der germanistischen Linguistik viel diskutiertes Problem. ${ }^{13}$ Die in Kontexten wie (3) und (4) auftretenden Schwankungen zwischen starker und schwacher Flexion (d.h. zwischen Parallelflexion und Wechselflexion) werden in der Literatur oft als Markiertheitsabbau gedeutet, der im Rahmen der voranschreitenden Tendenz zur Monoflexion diachron zum Verlust redundanter starker Flexive am Adjektiv führt.

a. einiges brauchbares Material

b. einiges brauchbare Material
a. aller guter Dinge
b. aller guten Dinge

Im Gegenwartsdeutschen führt dieser Sprachwandelprozess zu einem komplexen System sprachlicher Variation. Parallel- und Wechselflexion alternieren nicht beliebig, sondern es liegen abhängig von Typ und Form des Pronominaladjektivs von Fall zu Fall unterschiedliche Präferenzen für Parallel- bzw. Wechselflexion vor. Dabei treten die folgenden Variationsmuster auf (vgl. Wiese 2009). Zum einen

$13 \mathrm{Zu}$ den relevanten Entwicklungen im bzw. bis zum 20. Jahrhundert vgl. Paul (1919); Curme (1922); Behaghel (1923); Steche (1927); Wälterlin (1941); Törnqvist (1974); Solms/Wegera (1991); Voeste (2000); Schwerdt (2007); zum Gegenwartsdeutschen z.B. Ljungerud (1955); Dudengrammatik (1959, 2016); Zifonun et al. (1997); Marillier (2003); Sahel (2006); Wiese (2009). 
kann nach ein und derselben Form eines bestimmten Pronominaladjektivs ein nachfolgendes Adjektiv starke oder schwache Flexion tragen:

a. manche junge/jungen Männer (Nom./Akk. Pl.)

b. mancher junger/jungen Männer (Gen. Pl.)

Zum anderen kann man beobachten, dass nach unterschiedlichen Wortformen eines Pronominaladjektivs unterschiedliche Tendenzen zu starker bzw. schwacher Flexion am Adjektiv bestehen können. So wird bei einig- im Kontext Nom./ Akk. Pl. ein nachfolgendes Adjektiv bevorzugt stark flektiert, während bei dem gleichen Pronominaladjektiv im Dat. Sg. Mask./Neutr. eine Tendenz zur Wechselflexion besteht:

(6) a. einige junge Männer (Nom./Akk. Pl., stark)

b. einigem guten Willen (Dat. Sg., schwach)

Schließlich können auch bei Vorliegen identischer Flexionsmerkmale abhängig von der Wahl des Pronominaladjektivs unterschiedliche Verteilungen von starken und schwachen Formen auftreten. Während nach dem Nom./Akk. Pl. von welch- das Adjektiv quasi obligatorisch schwach flektiert wird, besteht im Nom./ Akk. Pl. von ander- eine Tendenz zur Parallelflexion:

a. welche jungen Männer (Nom./Akk. Pl., schwach)

b. andere junge Männer (Nom./Akk. Pl., stark)

Die Beschreibung und theoretische Deutung dieser empirischen Befunde ist notorisch schwierig. So stellt die Dudengrammatik (1959, S. 209) fest, dass „die Störungen im Deklinationssystem eine übersichtliche Zusammenfassung in Gruppen nicht recht zulassen“. Als Durchbruch kann daher die Arbeit von Wiese (2009) gewertet werden, dem es auf der Basis einer Sichtung der einschlägigen Literatur erstmals gelingt, eine Systematisierung der Schwankungen $\mathrm{zu}$ formulieren.

\subsubsection{Wiese (2009)}

Wieses Ansatz liegt die Erkenntnis zugrunde, dass die Adjektivflexion nicht beliebig alterniert; vielmehr lassen sich beim Übergang von Parallelflexion zu Wechselflexion nach Pronominaladjektiven bestimmte Muster erkennen, die auf das Wirken der folgenden beiden Faktoren zurückgeführt werden können (vgl. auch Dudengrammatik 2016, S. 960 ff.): 
- Lexembezogen: Je ähnlicher ein Pronominaladjektiv dem definiten Artikel ist, desto größer ist die Tendenz zur schwachen Flexion am Adjektiv; ${ }^{14}$

- Formbezogen: Der Übergang von starker zu schwacher Flexion erfolgt bevorzugt in markierten Flexionsformen (Markiertheitsabbau). ${ }^{15}$

Während der erste Faktor die Wahrscheinlichkeit beeinflusst, mit der nach einem bestimmten Pronominaladjektiv ein Adjektiv schwach flektiert wird, beschreibt der zweite Faktor den Weg, den die Ausbreitung der schwachen Flexion innerhalb eines Paradigmas nimmt - zunächst werden stärker markierte Formen wie Gen. Sg. Mask./Neutr. -s oder Dat. Sg. Mask./Neutr. - $m$ verdrängt, zuletzt schwächer markierte wie Nominativ Pl. -e. Zusammen ergeben die beiden Faktoren das Bild in Tabelle 1, in dem sich der Übergang von starker zu schwacher Flexion nach Pronominaladjektiven in verschiedenen Stufen vollzieht (also nicht zufällig verläuft), die sich als verschiedene Flexionstypen systematisieren lassen (vgl. Wiese 2009, S. 186). Die Hierarchisierung der Zeilen (die verschiedene Flexionstypen repräsentieren) ist dabei abhängig von lexikalischen Eigenschaften der jeweiligen Pronominaladjektive. Pronominaladjektive, die weiter oben angesiedelt sind,

14 Um die Nähe eines Pronominaladjektivs zum Prototyp des definiten Artikels zu bestimmen, wendet Wiese eine Reihe von Tests an, die die Syntax und Semantik der Pronominaladjektive betreffen. Generell gilt dabei: Je geringer der deskriptive Bedeutungsgehalt eines Pronominaladjektivs, desto größer die Nähe zum Artikel. So handelt es sich bei all- um einen reinen Quantor, während sämtlich- zusätzlich noch ausdrückt, dass es sich um alle Mitglieder einer Teilgruppe von Elementen handelt. Bei beid- liegt eine Kombination aus Allquantor und dem Zahlbegriff , $2^{\star}$ vor. Ein weiteres semantisches Kriterium wird aus dem Begriff der „Inklusivität“ abgeleitet. Dieser beruht auf der Beobachtung, dass sich Artikel und Allquantoren dahingehend ähnlich sind, dass sie (in einem bestimmten Kontext) auf eine Gesamtheit von Entitäten/Personen referieren können (im Gegensatz zu etwa Existenzquantoren), vgl. (i).

Peter war gestern auf einer Hochzeitsfeier. Die/Alle Brautjungfern trugen Rot.

Auf der Basis dieser semantischen Kriterien schließt Wiese, dass Allquantoren in der Regel artikelähnlicher sind als Existenzquantoren. Ein syntaktischer Test leitet sich ab aus dem sogenannten „NP-Bildungskriterium“ von Zifonun et al. (1997, S. 1929). Im Mittelpunkt steht dabei die Frage, ob sich das Pronominaladjektiv mit singularischen Individuativa zu einer vollständigen NP verbinden lässt. Wie in (ii) gezeigt, gilt dies zwar für typische Determinierer, aber nicht für das (relativ artikelähnliche) Element all-:

(ii) a. das/jedes Pferd

b. *alles Pferd

15 Nach Wiese (2009) ist die relative Markiertheit einer Flexionsform zum einen abhängig von ihrem Merkmalsgehalt (insbesondere der Kasushierarchie Genitiv > Dativ > Akkusativ > Nominativ) und zum anderen von der relativen konsonantischen Stärke des phonologischen Exponenten $(-e s>-e m>-e n>-e r>-e)$. 
stehen den Artikeln näher und lösen daher tendenziell eher schwache Flexion am nachfolgenden Adjektiv aus. Die Anordnung der Spalten ist abhängig von der relativen Markiertheit der Wortformen, die von links nach rechts zunimmt. Innerhalb eines Flexionstyps (d.h. einer Zeile) gilt, dass sich der Übergang von Parallelzu Wechselflexion von rechts nach links vollzieht, d.h., der Markiertheitsabbau erfasst zunächst markierte Wortformen.

Tab. 1: Verteilung starker und schwacher Adjektivflexion nach Pronominaladjektiven (nach Wiese 2009, S. 190)

\begin{tabular}{|c|c|c|c|c|c|c|}
\hline & $\begin{array}{l}\text { Nom./Akk. } \\
\text { Pl. } \\
(-e /-e n)\end{array}$ & $\begin{array}{l}\text { Gen. PI. } \\
\text { (-er/-en) }\end{array}$ & $\begin{array}{l}\text { Nom. Sg. } \\
\text { Mask. } \\
(-e r /-e)\end{array}$ & $\begin{array}{l}\text { Dat./Gen. } \\
\text { Sg. Fem. } \\
\text { (-er/-en) }\end{array}$ & $\begin{array}{l}\text { Nom./Akk. } \\
\text { Sg. Neutr. } \\
(-e s /-e)\end{array}$ & $\begin{array}{l}\text { Dat. Sg. } \\
\text { Mask./ } \\
\text { Neutr. } \\
\text { (-em/-en) }\end{array}$ \\
\hline all & schwach & schwach & schwach & schwach & schwach & schwach \\
\hline welch & $\begin{array}{l}\text { eher } \\
\text { schwach }\end{array}$ & $\begin{array}{l}\text { eher } \\
\text { schwach }\end{array}$ & schwach & schwach & schwach & schwach \\
\hline sämtlich & $\begin{array}{l}\text { eher } \\
\text { schwach }\end{array}$ & $\begin{array}{l}\text { eher } \\
\text { schwach }\end{array}$ & schwach & schwach & schwach & schwach \\
\hline beid & $\begin{array}{l}\text { eher } \\
\text { schwach }\end{array}$ & $\begin{array}{l}\text { eher } \\
\text { schwach }\end{array}$ & NA & NA & NA & NA \\
\hline solch & $\begin{array}{l}\text { eher } \\
\text { schwach }\end{array}$ & $\begin{array}{l}\text { eher } \\
\text { schwach }\end{array}$ & schwach & schwach & schwach & schwach \\
\hline manch & $\begin{array}{l}\text { eher } \\
\text { schwach/ } \\
\text { umstritten }\end{array}$ & $\begin{array}{l}\text { eher } \\
\text { schwach/ } \\
\text { umstritten }\end{array}$ & schwach & schwach & schwach & schwach \\
\hline folgend & eher stark & eher stark & schwach & schwach & schwach & schwach \\
\hline mehrer & stark & eher stark & NA & NA & NA & NA \\
\hline einig & stark & stark & $\begin{array}{l}\text { eher stark/ } \\
\text { umstritten }\end{array}$ & $\begin{array}{l}\text { eher stark/ } \\
\text { umstritten }\end{array}$ & schwach & schwach \\
\hline viel & stark & stark & $\begin{array}{l}\text { eher stark/ } \\
\text { umstritten }\end{array}$ & $\begin{array}{l}\text { eher stark/ } \\
\text { umstritten }\end{array}$ & schwach & schwach \\
\hline wenig & stark & stark & $\begin{array}{l}\text { eher stark/ } \\
\text { umstritten }\end{array}$ & $\begin{array}{l}\text { eher stark/ } \\
\text { umstritten }\end{array}$ & $\begin{array}{l}\text { eher stark/ } \\
\text { umstritten }\end{array}$ & schwach \\
\hline ander & stark & eher stark & $\begin{array}{l}\text { eher stark/ } \\
\text { umstritten }\end{array}$ & $\begin{array}{l}\text { eher stark/ } \\
\text { umstritten }\end{array}$ & $\begin{array}{l}\text { eher stark/ } \\
\text { umstritten }\end{array}$ & schwach \\
\hline
\end{tabular}


Dieses Modell erlaubt es, die Beobachtungen aus der einschlägigen Literatur systematisch zu erfassen (der Eintrag „umstritten“ signalisiert, dass sich die von Wiese konsultierten Grammatiken/Beschreibungen nicht einig sind). Zwischen zwei Polen, in denen kaum oder gar keine Variation vorliegt (wie dem Nom./ Akk. Pl. von ander- und dem Dat. Sg. Mask./Neutr. von all-) erfolgt ein gestaffelter Übergang von starken zu schwachen Endungen. Der Ansatz von Wiese macht dabei die folgenden Prognosen:

1. Innerhalb einer Spalte nimmt die Tendenz zu starker Flexion von oben nach unten $\mathrm{zu}$.

2. Innerhalb einer Zeile nimmt die Tendenz zu starker Flexion von rechts nach links zu.

Wir haben bereits erwähnt, dass es sich bei der empirischen Grundlage von Wiese (2009) in erster Linie um Grammatiken bzw. einschlägige Forschungsliteratur handelt, deren Urteile allerdings nicht immer übereinstimmen (,umstritten“ in Tab. 1). Um ein klareres Bild von den tatsächlichen Verhältnissen im Gegenwartsdeutschen $\mathrm{zu}$ bekommen, haben wir die Prognosen von Wieses Modell im Rahmen einer Korpusstudie überprüft.

\subsubsection{Befunde der Korpusuntersuchung}

Zunächst haben wir aus dem kleineren Teilkorpus (ca. 122 Mio. Tokens) 21.284 Belege für das Muster „Pronominaladjektiv-Adjektiv ( $\neq$ Superlativ $\left.^{16}\right)-$ Nomen“ extrahiert, durchgesehen und manuell für weitere Informationen wie starke vs. schwache Flexion, Flexionsmerkmale und Kasusregent annotiert. ${ }^{17}$ Für Wortformen, die in diesem kleineren Teilkorpus nicht oder nur in $\mathrm{zu}$ geringer Zahl auffindbar waren, haben wir zusätzlich Daten aus dem ca. 7 Mrd. Tokens umfassenden Ausschnitt aus DEREKo nacherhoben. ${ }^{18}$ Die Befunde sind in Tabelle 2 zusammengefasst. Es lässt sich bereits auf den ersten Blick erkennen, dass die auf Korpusbefunden

16 Der Superlativ wurde ausgeschlossen, um bei der Suche nach der Form aller keine Fehlbelege der Form das aller schönste Ferienerlebnis zu erhalten.

17 In Fällen, in denen die Belegzahl die Schwelle von 500 Beispielen deutlich überstieg, haben wir mindestens 300 Belege gesichtet und das Ergebnis auf die Gesamtheit der Daten hochgerechnet.

18 Dabei handelte es sich um folgende Fälle: manch-/viel-/welch-/wenig- (Nom. Sg. Mask., stark/ schwach), einig-/manch-/viel-/welch-/wenig- (Dat. Sg. Fem., stark/schwach), viel- (Nom./Akk. Sg. Neutr., schwach; Dat. Sg. Mask./Neutr., stark/schwach), wenig- (Dat. Sg. Mask./Neutr., stark/ schwach) sowie folgend- (Gen. Pl., stark/schwach). 
basierende Tabelle nicht mehr die Unsicherheiten aufweist, die bei Wiese auf die unterschiedlichen Einschätzungen verschiedener Grammatiken zurückgehen (,umstrittene“ Positionen). ${ }^{19}$

Tab. 2: Anteil starker Flexionsformen nach Markiertheit der Flexionsendung und Pronominaladjektiv (Ausschnitt aus DEREKo)

\begin{tabular}{|c|c|c|c|c|c|c|}
\hline & $\begin{array}{l}\text { Nom./Akk. } \\
\text { Pl. } \\
(-e /-e n)\end{array}$ & $\begin{array}{l}\text { Gen. PI. } \\
\text { (-er/-en) }\end{array}$ & $\begin{array}{l}\text { Nom. Sg. } \\
\text { Mask. } \\
(-e r /-e)\end{array}$ & $\begin{array}{l}\text { Dat./Gen. } \\
\text { Sg. Fem. } \\
\text { (-er/-en) }\end{array}$ & $\begin{array}{l}\text { Nom./Akk. } \\
\text { Sg. Neutr. } \\
\text { (-es/-e) }\end{array}$ & $\begin{array}{l}\text { Dat. Sg. } \\
\text { Mask./ } \\
\text { Neutr. } \\
\text { (-em/-en) }\end{array}$ \\
\hline all & $1 \%$ & $5 \%$ & $58 \%$ & $13 \%$ & $1 \%$ & $5 \%$ \\
\hline welch & $6 \%$ & $0 \%$ & $4 \%$ & $4 \%$ & $4 \%$ & $0 \%$ \\
\hline sämtlich & $24 \%$ & $88 \%$ & $93 \%$ & $85 \%$ & $71 \%$ & $9 \%$ \\
\hline beid & $33 \%$ & $49 \%$ & NA & NA & NA & NA \\
\hline solch & $8 \%$ & $42 \%$ & $91 \%$ & $36 \%$ & $64 \%$ & $11 \%$ \\
\hline manch & $56 \%$ & $82 \%$ & $12 \%$ & $24 \%$ & $5 \%$ & $0 \%$ \\
\hline folgend & $86 \%$ & $98 \%$ & $97 \%$ & $68 \%$ & $65 \%$ & $12 \%$ \\
\hline mehrer & $99 \%$ & $99 \%$ & NA & NA & NA & NA \\
\hline einig & $99 \%$ & $98 \%$ & $100 \%$ & $96 \%$ & $79 \%$ & $14 \%$ \\
\hline viel & $99 \%$ & $98 \%$ & NA & $100 \%$ & $54 \%$ & $21 \%$ \\
\hline wenig & $100 \%$ & $100 \%$ & $100 \%$ & $100 \%$ & $100 \%$ & $40 \%$ \\
\hline ander & $99 \%$ & $98 \%$ & $100 \%$ & $99 \%$ & $95 \%$ & $23 \%$ \\
\hline
\end{tabular}

19 Allerdings muss angemerkt werden, dass Fälle, in denen starke und schwache Flexion annähernd gleich verteilt sind (wie der Gen. Pl. von beid-), weiterhin schwierig zu interpretieren sind. 
In Übereinstimmung mit Wiese (2009) lösen Pronominaladjektive wie all- und welch-, die nach allgemeiner Auffassung Determinierern ähnlich sind, überwiegend schwache Flexion an einem nachfolgenden Adjektiv aus, wobei ein deutlicher Kontrast zu anderen Pronominaladjektiven vorliegt (insbesondere am unteren Tabellenende dominiert starke Flexion am Adjektiv). Ebenfalls im Einklang mit Wiese (2009) erweist sich die Merkmalskombination Dat. Sg. Mask./Neutr. als bevorzugtes Einfallstor für die schwache Flexion. Wesentlich auffälliger sind allerdings die Abweichungen von der nach Wiese (2009) erwarteten Verteilung der Flexionsvarianten. Generell treten mehr starke Endungen auf, d.h., das Niveau der starken Formen liegt insgesamt wesentlich höher als prognostiziert. Zudem zeigt sich im Übergangsbereich nicht der erwartete gestaffelte bzw. stufenförmige Anstieg der starken Endungen von rechts unten nach links oben. Stattdessen erscheint im mittleren Bereich der Markiertheitsskala ein „Berg“, d.h. eine Häufung starker Endungen, insbesondere im Zusammenhang mit Nom. Sg. Mask. (-er/-e) (mit der Ausnahme von welch-). Diese etwas überraschenden Ergebnisse der Korpusstudie werfen eine Reihe von Fragen auf. Im weiteren Verlauf wollen wir uns vor allem mit den folgenden beiden Problemen befassen:

1. Wie lässt sich das im Vergleich zu Wiese (2009) insgesamt höhere Niveau starker Endungen interpretieren?

2. Inwiefern lassen sich die Korpusbefunde mit Wieses (2009) Annahme eines gestaffelten Markiertheitsabbaus vereinbaren?

Der Erklärungsansatz, den wir dabei verfolgen wollen, basiert auf der Annahme, dass die Abweichungen von der erwarteten Verteilung der Reflex eines noch nicht abgeschlossenen Sprachwandelprozesses sind, der in Teilen der Ausbreitung der schwachen Flexion entgegenläuft.

\subsubsection{Sprachwandel?}

Ausgangspunkt unserer Überlegungen ist die Beobachtung, dass die Datengrundlage von Wiese (2009) in erster Linie aus grammatischen Beschreibungen des Deutschen in der Mitte des 20. Jahrhunderts besteht (wie Wälterlin 1941; Ljungerud 1955; Dudengrammatik 1959; Hansen 1963; Pfeffer/Linder 1984). Darauf aufbauend möchten wir die Hypothese vertreten, dass Wieses Analyse zwar eine zutreffende Beschreibung dieses (historischen) grammatischen Systems liefert, dass aber aufgrund von relativ rezenten (und noch nicht abgeschlossenen) Sprachwandelprozessen eine Anpassung des Modells nötig ist. Um diese Annahme zu stützen, haben wir eine Korpusstudie im historischen Korpus HIST durchgeführt, das Texte von 1700 bis etwa 1918 umfasst (Schwerpunkt: 19. Jahrhundert). Für 
unsere Untersuchungen haben wir einen ca. 30 Mio. Tokens umfassenden Teil von HIST geparst und mit zusätzlichen morphosyntaktischen Annotationen versehen. Die Ergebnisse der Untersuchung sind in Tabelle 3 abgelegt.

Tab. 3: Anteil starker Flexionsformen nach Markiertheit der Flexionsendung und Pronominaladjektiv (Untersuchungskorpus HIST)

\begin{tabular}{|c|c|c|c|c|c|c|}
\hline & $\begin{array}{l}\text { Nom./Akk. Pl. } \\
(-e /-e n)\end{array}$ & $\begin{array}{l}\text { Gen. PI. } \\
\text { (-er/-en) }\end{array}$ & $\begin{array}{l}\text { Nom. Sg. } \\
\text { Mask. } \\
(-e r /-e)\end{array}$ & $\begin{array}{l}\text { Dat./Gen. } \\
\text { Sg. Fem. } \\
\text { (-er/-en) }\end{array}$ & $\begin{array}{l}\text { Nom./Akk. } \\
\text { Sg. Neutr. } \\
(-e s /-e)\end{array}$ & $\begin{array}{l}\text { Dat. Sg. } \\
\text { Mask./ } \\
\text { Neutr. } \\
\text { (-em/-en) }\end{array}$ \\
\hline all & $\begin{array}{l}24 \% \\
(878 / 3646)\end{array}$ & $\begin{array}{l}<1 \% \\
(9 / 1280)\end{array}$ & $\begin{array}{l}14 \% \\
(8 / 57)\end{array}$ & $\begin{array}{l}1 \% \\
(11 / 807)\end{array}$ & $\begin{array}{l}<1 \% \\
(2 / 308)\end{array}$ & $\begin{array}{l}3 \% \\
(7 / 217)\end{array}$ \\
\hline welch & $\begin{array}{l}58 \% \\
(130 / 225)\end{array}$ & $\begin{array}{l}0 \% \\
(0 / 8)\end{array}$ & $\begin{array}{l}0 \% \\
(0 / 48)\end{array}$ & $\begin{array}{l}2 \% \\
(3 / 147)\end{array}$ & $\begin{array}{l}0 \% \\
(0 / 64)\end{array}$ & $\begin{array}{l}2 \% \\
(2 / 107)\end{array}$ \\
\hline sämtlich & $\begin{array}{l}64 \% \\
(29 / 45)\end{array}$ & $\begin{array}{l}33 \% \\
(6 / 18)\end{array}$ & NA & NA & NA & NA \\
\hline beid & $\begin{array}{l}81 \% \\
(59 / 73)\end{array}$ & $\begin{array}{l}33 \% \\
(9 / 27)\end{array}$ & NA & NA & NA & NA \\
\hline solch & $\begin{array}{l}88 \% \\
(540 / 613)\end{array}$ & $\begin{array}{l}18 \% \\
(35 / 199)\end{array}$ & $\begin{array}{l}50 \% \\
(6 / 12)\end{array}$ & $\begin{array}{l}9 \% \\
(14 / 157)\end{array}$ & $\begin{array}{l}37 \% \\
(7 / 19)\end{array}$ & $\begin{array}{l}4 \% \\
(3 / 73)\end{array}$ \\
\hline manch & $\begin{array}{l}93 \% \\
(187 / 202)\end{array}$ & $\begin{array}{l}18 \% \\
(9 / 51)\end{array}$ & $\begin{array}{l}17 \% \\
(19 / 111)\end{array}$ & $\begin{array}{l}1 \% \\
(1 / 89)\end{array}$ & $\begin{array}{l}5 \% \\
(8 / 153)\end{array}$ & $\begin{array}{l}3 \% \\
(2 / 68)\end{array}$ \\
\hline folgend & $\begin{array}{l}100 \% \\
(53 / 53)\end{array}$ & NA & $\begin{array}{l}60 \% \\
(3 / 5)\end{array}$ & $\begin{array}{l}50 \% \\
(7 / 14)\end{array}$ & $\begin{array}{l}15 \% \\
(3 / 20)\end{array}$ & $\begin{array}{l}8 \% \\
(1 / 13)\end{array}$ \\
\hline mehrer & $\begin{array}{l}99 \% \\
(356 / 358)\end{array}$ & $\begin{array}{l}50 \% \\
(37 / 74)\end{array}$ & NA & NA & NA & NA \\
\hline einig & $\begin{array}{l}99 \% \\
(1219 / 1222)\end{array}$ & $\begin{array}{l}38 \% \\
(67 / 177)\end{array}$ & NA & $\begin{array}{l}44 \% \\
(8 / 18)\end{array}$ & $\begin{array}{l}46 \% \\
(6 / 13)\end{array}$ & $\begin{array}{l}9 \% \\
(1 / 11)\end{array}$ \\
\hline viel & $\begin{array}{l}99 \% \\
(947 / 958)\end{array}$ & $\begin{array}{l}45 \% \\
(101 / 224)\end{array}$ & NA & $\begin{array}{l}78 \% \\
(7 / 9)\end{array}$ & $\begin{array}{l}75 \% \\
(3 / 4)\end{array}$ & $\begin{array}{l}6 \% \\
(1 / 17)\end{array}$ \\
\hline wenig & $\begin{array}{l}100 \% \\
(89 / 89)\end{array}$ & $\begin{array}{l}70 \% \\
(7 / 10)\end{array}$ & NA & NA & $\begin{array}{l}100 \% \\
(1 / 1)\end{array}$ & NA \\
\hline ander & $\begin{array}{l}99 \% \\
(610 / 618)\end{array}$ & $\begin{array}{l}76 \% \\
(145 / 190)\end{array}$ & $\begin{array}{l}100 \% \\
(4 / 4)\end{array}$ & $\begin{array}{l}92 \% \\
(11 / 12)\end{array}$ & $\begin{array}{l}100 \% \\
(6 / 6)\end{array}$ & $\begin{array}{l}27 \% \\
(3 / 11)\end{array}$ \\
\hline
\end{tabular}

Aufgrund des im Vergleich zu den anderen verwendeten Korpora relativ geringen Umfangs von HIST sind die Belegzahlen zum Teil recht klein (in Klammern hinter 
den Prozentzahlen); zudem gibt es insbesondere bei den markierteren/selteneren Merkmalskombinationen mehr Lücken in der Tabelle. Dennoch ist es augenfällig, dass die Resultate besser in Einklang stehen mit den Prognosen von Wiese (2009) als die Befunde zur Gegenwartssprache. Es liegt insgesamt ein geringerer Anteil starker Endungen vor; auch der gestaffelte, stufenförmige Verlauf des Übergangs von starker zu schwacher Flexion ist wesentlich besser erkennbar. Allerdings ist bereits in den Daten aus HIST in Ansätzen der „Berg“ im Bereich Nom. Sg. Mask. erkennbar, der im Gegenwartsdeutschen noch stärker zutage tritt.

Ein Vergleich der beiden Studien zeigt, dass man im Bereich der Adjektivflexion nach Pronominaladjektiven offenbar zwei (teilweise) gegenläufige Sprachwandelprozesse unterscheiden muss. Dem Markiertheitsabbau vor allem im Bereich Dat. Sg. Mask./Neutr. steht eine generelle Ausbreitung der starken Flexion nach Pronominaladjektiven entgegen (vgl. bereits van Dam 1963, S. 280 f.). Bei letzterer Entwicklung handelt es sich offenbar um einen rezenten Sprachwandelprozess, der möglicherweise erst in den letzten 50 bis 100 Jahren Fahrt aufgenommen hat und in den von Wiese konsultierten Grammatiken aus der Mitte des 20. Jahrhunderts noch nicht registriert wurde. Zusammengenommen scheinen diese beiden Prozesse das Flexionsverhalten in Kombinationen aus Pronominaladjektiv + Adjektiv an das Flexionsverhalten in Adjektivreihungen anzupassen (möglicherweise handelt es sich also um einen analogischen Sprachwandel): Wechselflexion erfolgt in erster Linie im Kontext Dat. Sg. Mask./Neutr.; in allen anderen Fällen liegt eine Tendenz zur Parallelflexion vor. Eine Ausnahme bilden die Pronominaladjektive all- und welch-, die den Determinierern nahestehen und daher in der Regel Wechselflexion auslösen. Auffällig ist ferner, dass die Ausbreitung der starken Flexion im Kontext Nom. Sg. Mask. besonders stark fortgeschritten ist. Dies kann als Hinweis darauf verstanden werden, dass es sich hierbei um den unmarkierten Fall (unmarkierte Merkmalskombination + relativ leichtes Flexiv -er/-e) handelt und dass diachron die Verteilung der (starken) Endungen im Sinne der Markiertheitshierarchie neu geordnet wird - bereits Wiese (2009, S. 187) stellt fest, dass in unmarkierten Positionen des Paradigmas die Verdrängung der schwachen durch die starke Flexion am wahrscheinlichsten ist. Um die Ausbreitung der starken Flexion auf diese Weise zu erfassen, ist es allerdings erforderlich, das Modell von Wiese an die empirischen Befunde anzupassen. Diese Modifikation ist zurzeit in Arbeit und soll Eingang finden in die Grammatik des Deutschen, die gegenwärtig im Rahmen des Projekts „Korpusgrammatik“ am IDS entsteht. 


\section{Zusammenfassung}

Die Stark/schwach-Alternation der Adjektivflexion wird von mehreren Faktoren beeinflusst, deren Zusammenspiel komplexe Muster sprachlicher Variation hervorbringt. Die beiden Endpunkte des Kontinuums sind zum einen die Kombination aus flektiertem Determinierer + Adjektiv, bei der ausschließlich Wechselflexion (stark/schwach) vorliegt. Am anderen Ende der Skala liegen Adjektivreihungen (ohne Determinierer), die in der Regel Parallelflexion (stark/stark) zeigen. Die Ausnahme bildet die markierte Kombination Dat. Sg. Mask./Neutr., bei der die Wechselflexion auch bei Adjektivfolgen ohne Determinierer in Deutschland und in Österreich die häufigste Variante darstellt. Dabei haben wir im Rahmen eines explorativen korpusbasierten Zugangs zunächst weitere Einflussgrößen identifiziert, die auf die Wahl zwischen Parallel- und Wechselflexion wirken. Zwischen den o.g. Polen bewegen sich Pronominaladjektive. Hier ist die Wahl zwischen starker und schwacher Flexion an nachfolgenden Adjektiven abhängig von (i) Eigenschaften des Pronominaladjektivs (Nähe zum Artikel) und (ii) Eigenschaften der Flexionsform (relative Markiertheit), vgl. Wiese (2009). Wir haben in diesem Beitrag gezeigt, dass sich im Gegensatz zur herrschenden Meinung die Parallelflexion (stark/stark) in diesem Kontext ausbreitet. Dies kann so gedeutet werden, dass sich das Flexionsverhalten in Fügungen aus Pronominaladjektiv und Adjektiv an die Gegebenheiten bei Adjektivreihungen ohne Determinierer bzw. bei Kombinationen aus Determinierer und Adjektiv (welch- und all-) angleicht. Der Markiertheitsabbau ist (analog zu Adjektivreihungen) primär auf den Kontext Dat. Sg. Mask./Neutr. beschränkt.

\section{Literatur}

Adelung, Johann Christoph (1782/2010): Umständliches Lehrgebäude der Deutschen Sprache. Zur Erläuterung der Deutschen Sprachlehre für Schulen. Aachen. Internet: http://nbnresolving.de/urn:nbn:de:S2w-568 (Stand: 12.12.2017).

Behaghel, Otto (1923): Deutsche Syntax. Eine geschichtliche Darstellung. Bd. I: Die Wortklassen und Wortformen. A: Nomen. Pronomen. Heidelberg.

Belica, Cyril et al. (2012): DeReWo. Korpusbasierte Grund-/Wortformenlisten. Derewo-v-ww-bll320000g-2012-12-31-1.0. Internet: www1.ids-mannheim.de/kl/projekte/methoden/ derewo.html (Stand: 26.7.2017).

Berk, Richard A./Freedman, David A. (2009): Statistical assumptions as empirical commitments. In: Collier, David/Sekhon, Jasjeet S./Stark, Philip B. (Hg.): Statistical models and causal inference. A dialogue with the social sciences. Cambridge, S. 23-44.

Berk, Richard/Brown, Lawrence/Zhao, Linda (2010): Statistical inference after model selection. In: Journal of Quantitative Criminology 26, S. 217-236. 
Bubenhofer, Noah/Konopka, Marek/Schneider, Roman (2014): Präliminarien einer Korpusgrammatik. (= Korpuslinguistik und interdisziplinäre Perspektiven auf Sprache 4). Tübingen. [Unter Mitarbeit von Caren Brinckmann, Katrin Hein und Bruno Strecker].

Cohen, Ayala (1980): On the graphical display of the significant components in two-way contingency tables. In: Communications in Statistics. Theory and Methods 9, 10, S. 1025-1041. DOI: $10.1080 / 03610928008827940$.

Curme, George O. (1922): A grammar of the German language. 2., überarb. Aufl. New York.

Dudengrammatik (1959) = Dudenredaktion (1959) (Hg.): Der große Duden. Bd. 4: Grammatik der deutschen Gegenwartssprache. Völlig neu bearb. Aufl. Mannheim.

Dudengrammatik (2016) = Dudenredaktion (2016) (Hg.): Duden - Deutsche Sprache in 12 Bänden. Bd. 4: Die Grammatik. Unentbehrlich für richtiges Deutsch. 9., vollst. überarb. u. akt. Aufl. Berlin.

Eichinger, Ludwig M. (1987): Die Adjektive auf -isch und die Serialisierungsregeln in deutschen Nominalgruppen. In: Brekle, Herbert E./Asbach-Schnitker, Brigitte/Roggenhofer, Johannes (Hg.): Neuere Forschungen zur Wortbildung und Historiographie der Linguistik. Festgabe für Herbert E. Brekle zum 50. Geburtstag. (= Tübinger Beiträge zur Linguistik 284). Tübingen, S. 155-176.

Eichinger, Ludwig M. (1991): Ganz natürlich - aber im Rahmen bleiben. Zur Reihenfolge gestufter Adjektivattribute. In: Deutsche Sprache 4, S. 312-329.

Friendly, Michael (1992): Graphical methods for categorical data. In: SAS SUGI 17 Conference, April 1992. Internet: www.math.yorku.ca/SCS/sugi/sugi17-paper.html (Stand 25.1.2017). [Referat auf der SAS SUGI 17 Conference, April 1992].

Gallmann, Peter (2016): Die m-Verschwörung. Zum Abbau der starken Deklinationsendung -em. Internet: www.personal.uni-jena.de/ x1gape/Wort/Wort_NP_m_Schwaeche.pdf (Stand: 21.12.2016).

Hansen, Thorolf (1963): Zur Flexion der Adjektive nach Pronominaladjektiven in der deutschen Literatursprache nach 1945. In: Deutschunterricht für Ausländer 1, S. 129-137.

Heuer, Walter/Flückiger, Max/Gallmann, Peter (2017): Richtiges Deutsch. Vollständige Grammatik und Rechtschreiblehre. 32. Aufl. Zürich.

Kubczak, Jacqueline (2015): Mit echtem bayerischem Senf oder mit echtem bayerischen Senf? Flexion bei artikellosen Folgen von Adjektiven (aus „Grammatik in Fragen und Antworten“). In: Sprachreport 31, 3, S. 22-27. Internet: https://ids-pub.bsz-bw.de/files/4257/Kubczak _Mit_echtem_bayerischem_2015_3.pdf(Stand: 21.12.2016).

Kupietz, Marc et al. (2010): The German reference corpus DeReKo. A primordial sample for linguistic research. In: Calzolari, Nicole et al. (Hg.): Proceedings of the Seventh International Conference on Language Resources and Evaluation (LREC '10). Valletta, S. 1848-1854.

Ljungerud, Ivar (1955): Zur Nominalflexion in der deutschen Literatursprache nach 1900. Lund/ Kopenhagen.

Marillier, Jean-Francois (2003): Die Deklination der Determinative und Adjektive. Stellungsprinzip vs. Klassenprinzip. In: Baudot, Daniel/Behr, Irmtraud (Hg.): Funktion und Bedeutung. Modelle einer syntaktischen Semantik des Deutschen. Festschrift für Francois Schanen. (= Eurogermanistik 20). Tübingen, S. 75-94.

Meyer, David/Zeileis, Achim/Hornik, Kurt (2005): The Strucplot framework. Visualizing multi-way contingency tables with vcd. Internet: http://epub.wu.ac.at/480/ (Stand: 25.1.2017).

Moulin, Claudine (2000): Varianz innerhalb der Nominalgruppenflexion. Ausnahmen zur sogenannten Parallelflexion der Adjektive im Neuhochdeutschen. In: Germanistische Mitteilungen 52, S. 73-97. 
Niebuhr, Astrid (2017): Mit einem stetigem Anstieg. Variation in der Adjektivflexion. In: 39. Jahrestagung der Deutschen Gesellschaft für Sprachwissenschaft: Informatik \& sprachliche Kodierung. 8.-10. März 2017, Universität des Saarlandes. Saarbrücken. Nübling, Damaris (2011): Unter großem persönlichem oder persönlichen Einsatz? Der sprachliche Zweifelsfall adjektivischer Parallel- vs. Wechselflexion als Beispiel für aktuellen grammatischen Wandel. In: Köpcke, Klaus-Michael/Ziegler, Arne (Hg.): Grammatik. Lehren, Lernen, Verstehen. Zugänge zur Grammatik des Gegenwartsdeutschen. (= Germanistische Linguistik 293). Berlin, S. 175-195. Internet: http:// publikationen.ub.uni-frankfurt.de/frontdoor/index/index/docld/38277 (Stand: 21.12.2016). Paul, Hermann (1919): Deutsche Grammatik. Bd. 3/Teil 4: Syntax (1. Hälfte). Halle (Saale).

Peter, Klaus (2013): Steuerungsfaktoren für Parallel- und Wechselflexion in Adjektivreihungen. In: Jahrbuch für Germanistische Sprachgeschichte 4, 1, S. 186-204. Internet: https://www. degruyter.com/downloadpdf/j/jbgsg.2013.4.issue-1/jbgsg-2013-0012/jbgsg-2013-0012.xml (Stand: 12.1.2017).

Pfeffer, J. Alan/Linder, Beth (1984): The inflection of adjectives after indefinites in written and spoken German. In: Pfeffer, J. Alan (Hg.): Studies in descriptive German grammar. (= Studies in Descriptive Linguistics 12). Heidelberg, S. 135-162.

Rat für deutsche Rechtschreibung (2018): Deutsche Rechtschreibung. Regeln und Wörterverzeichnis. Mannheim. Internet: www.rechtschreibrat.com/DOX/rfdr_Regeln_2016_ redigiert_2018.pdf (Stand: 28.3.2018).

Sahel, Said (2006): Die Variation der Adjektivflexion nach Pronominaladjektiven und einige ihrer Determinanten. Eine empirische Untersuchung. In: Deutsche Sprache 33, S. 355381.

Schäfer, Roland (2017): Parallel- und Wechselflexion in obliken Adjektivreihen - eine empirische Vervollständigung. Vortrag am Institut für Deutsche Sprache, Mannheim, 31.5.2017.

Schmid, Helmut (1995): Improvements in part-of-speech tagging with an application to German. In: Proceedings of the EACL SIGDAT-Workshop. Dublin, S. 47-50.

Schmid, Helmut/Fitschen, Arne/Heid, Ulrich (2004): SMOR. A German computational morphology covering derivation, composition, and inflection. In: Lino, Maria T. et al. (Hg.): Proceedings of the IVth International Conference on Language Resources and Evaluation (LREC 2004). Paris, S. 1263-1266.

Schwerdt, Judith (2007): Die Flexion des attributiven Adjektivs im Mittelhochdeutschen. In: Sprachwissenschaft 32, S. 77-97.

Selvin, Hanan C./Stuart, Alan (1966): Data-dredging procedures in survey analysis. In: The American Statistician 20, 3, S. 20-23.

Solms, Hans-Joachim/Wegera, Klaus-Peter (1991): Grammatik des Frühneuhochdeutschen. Bd. 6: Flexion der Adjektive. Heidelberg.

Steche, Theodor (1927): Die neuhochdeutsche Wortbiegung unter besonderer Berücksichtigung der Sprachentwicklung im 19. Jahrhundert. Bd. 1: Die Wortklassen. Die Beugung. Breslau.

Törnqvist, Nils (1974): Zur Geschichte der deutschen Adjektivflexion. In: Neuphilologische Mitteilungen 75, S. 317-331.

Trost, Igor (2006): Das deutsche Adjektiv. Untersuchungen zur Semantik, Komparation, Wortbildung und Syntax. (= Beiträge zur germanistischen Sprachwissenschaft 19). Hamburg. [Diss. Passau 2005].

van Dam, J. (1963): Handbuch der deutschen Sprache. Bd. 2: Wortlehre. Groningen.

Voeste, Anja (2000): Varianz und Vertikalisierung. Zur Normierung der Adjektivdeklination in der ersten Hälfte des 18. Jahrhunderts. Amsterdam. 
Wälterlin, Kurt (1941): Die Flexion des Adjektivs hinter Formwörtern in der neueren deutschsprachigen Presse. Zürich.

Wiese, Bernd (2009): Variation in der Flexionsmorphologie. Starke und schwache Adjektivflexion nach Pronominaladjektiven. In: Konopka, Marek/Strecker, Bruno (Hg.): Deutsche Grammatik. Regeln, Normen, Sprachgebrauch. (= Jahrbuch des Instituts für Deutsche sprache 2008). Berlin/New York, S. 166-194.

Zifonun, Gisela et al. (1997): Grammatik der deutschen Sprache. (= Schriften des Instituts für Deutsche Sprache 7). Berlin/New York. 